\title{
Titanium Mesh for Bone Augmentation in Oral Implant Surgery
}

\author{
Youngdeok Chee, DDS, MSD, PhD* \\ Professor, Department of Oral \& Maxillofacial Surgery, College of Dentistry, Wonkwang University, Iksan, Korea \\ *Corresponding author: Youngdeok Chee, Department of Oral \& Maxillofacial Surgery, Sanbone dental \\ hospital, College of Dentistry, Wonkwang University, 1142, Sanbon-dong, Gunpo-city, Gyeonggi-do \\ 15865, Korea \\ Tel : +82-31-390-2875. Fax : +82-31-390-2777. E-mail : omschee@wku.ac.kr
}

OPEN ACCESS

pISSN 2765-7833

eISSN 2765-7841

Journal of implantology and applied sciences 2021; 25(4): 188-200

https://doi.org/10.32542/implantology.2021020

Received: October 5, 2021

Revised: December 5, 2021

Accepted: December 10, 2021

ORCID

Youngdeok Chee

http://orcid.org/0000-0002-0333-4685

Copyright $($ 2021. The Korean Academy of Oral \& Maxillofacial Implantology

This is an Open Access article distributed under the terms of the Creative Commons Attribution Non-Commercial License (http://creativecommons. org/licenses/by-nc/4.0/) which permits unrestricted non-commercial use, distribution, and reproduction in any medium, provided the original work is properly cited.

\begin{abstract}
Bone augmentation should be performed to recover a sufficient amount of bone before implant placement at the site where a bone defect occurs due to bone resorption caused by tooth extraction or periodontitis. Currently, bone augmentation through guided bone regeneration is widely used at the site of bone defects. However, after guided bone regeneration, the bone cannot maintain its shape when the procedure is performed at the site of a vertical bone defect. In such a case, bone augmentation for the desired shape can be performed using a titanium-reinforced membrane or titanium mesh to maintain the space three-dimensionally and create a bone shape at the site of the vertical bone defect. Titanium mesh, with its superior mechanical properties, prevents the collapse of the shape at the bone transplantation site, and enhances the stabilization of the wound by maintaining the space, enhancing the effect of bone regeneration, and contributing to the morphological enhancement of the regenerated alveolar bone. In this case, after bone augmentation was performed using titanium mesh at the site where the bone defect was large or a vertical bone defect occurred, the implant was placed, which led to a good outcome.
\end{abstract}

Keywords: Dental implant, Guided bone regeneration, Titanium mesh

\section{Introduction}

Currently, prosthetic restoration using an implant at the site of tooth loss is widely performed, and for successful implant surgery, a sufficient amount of bone is needed at the implant site. ${ }^{1}$ However, if tooth extraction due to severe periodontitis or trauma was previously performed, a defect or recession of alveolar bone occurs, making the placement of the implant; thus, various types of alveolar bone regeneration are required. ${ }^{2}$ Additionally, for the long-term stable prognosis of the implant, an appropriate amount of good quality bone is needed around the implant.

Nyman et al. ${ }^{3}$ introduced guided tissue regeneration, which uses a barrier membrane on the defect site in periodontal treatment. A further application of this technique, guided 
bone regeneration (GBR), has been introduced and widely used in bone augmentation for the placement of an implant. ${ }^{4}$

Thus, to place an implant on a bone defect site, bone augmentation is needed, and GBR is among the most well-known bone augmentation techniques. Other methods include alveolar ridge splitting and bone graft block. ${ }^{5}$ GBR is simple compared to other bone augmentation techniques; thus, it is often used when a bone graft is needed for the implant. GBR is a surgical method that creates a space with a membrane around the bone with insufficient height and width and thus induces the differentiation and migration of cells with the ability to form bone in the residual bone tissue, eventually regenerating the bone.

However, it is difficult to maintain the shape with GBR when bone augmentation is performed on the site where the bone defect occurs vertically, and thus, sometimes a tissue adhesive is additionally used to shape the graft material into a lump to fit the bone defect site.

Furthermore, when it is difficult to maintain the shape only with a tissue adhesive in bone augmentation, a titanium-reinforced Gore-Tex membrane or titanium mesh, which maintains the space threedimensionally and the shape of the membrane as desired, can be used for bone augmentation on the horizontal and vertical defect sites. Tissue adhesives or titanium-reinforced Gore-Tex membranes are not sufficient for reliable space maintenance, and it is more advantageous to use titanium mesh in bone augmentation to maintain the desired shape and restore the defective bone shape.

In this case study, it was difficult to perform bone augmentation in patients owing to the occurrence of horizontal and vertical bone defects. Thus, bone augmentation using GBR with titanium mesh was performed to form a sufficient amount of bone, and the implants were then placed. This led to good outcomes; thus, herein, we report these cases.

\section{II . Case Report}

\section{Case 1}

A 57-year-old female patient was referred to our hospital for bone graft and implant placement due to a severe bone defect after extraction of the right mandibular first molar at a local dental clinic. As severe horizontal and vertical bone defects were observed on the panoramic radiograph, and the distance to the right mandibular inferior alveolar nerve was short, delayed implant surgery after vertical and horizontal bone augmentation using titanium mesh was planned (Fig. 1).

After local anesthesia (2\% lidocaine with 1:100,000 epinephrine; Yuhan, Seoul, Korea) was administered on the site where the right mandibular first molar had been extracted, a vertical incision 
was made on both sides of the surgical site, and after crestal incision, a full-thickness flap was elevated (Fig. 2A, 2B). After flap elevation, a vertical bone defect (2-3 mm) was observed, and thus, bone grafting was performed on the bone defect site using $0.5 \mathrm{cc}$ of allograft bone (Oragraft ${ }^{\circledR}$; LifeNet, Virginia, USA) with a tissue adhesive, fibrin glue (Tisseel ${ }^{\mathbb{\circledR}}$; Baxter, Vienna, Austria) (Fig. 2C). For this, titanium mesh (size $34 \times 25 \mathrm{~mm}$ ) (Jeil Medical, Seoul, Korea) was used to shape the bone, maintain the bone shape at the defect site, and two micro screws were used to fix the titanium mesh at the bottom (anterior and posterior) (Fig. 2D). After surgery, the bone graft and titanium mesh were observed to be

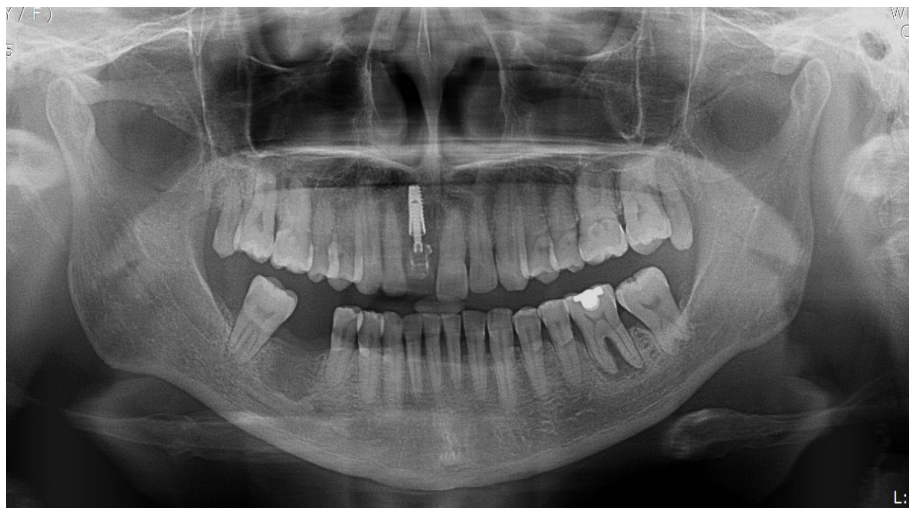

Fig. 1. Case I: Pre-operative panoramic view.
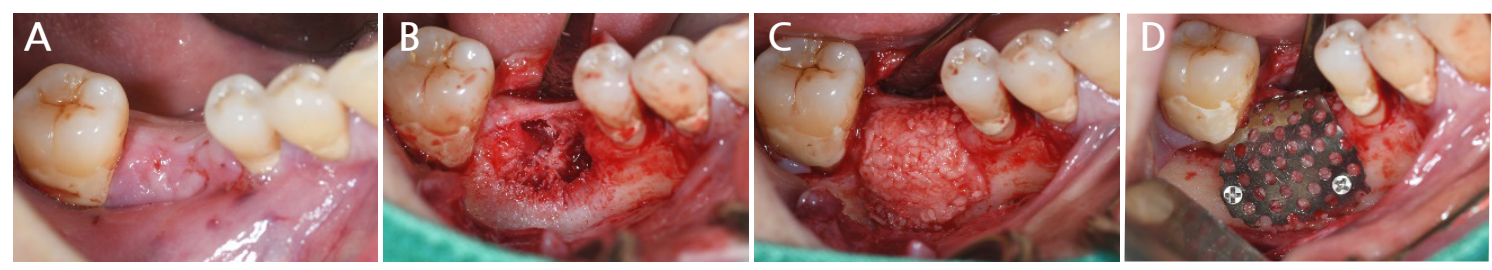

Fig. 2. Case I: Intraoral views of the bone graft with titanium mesh. (A) Pre-operative intraoral view, (B) A full-thickness flap is elevated, (C) Bone grafting using allograft, (D) Application of titanium mesh with micro screws.

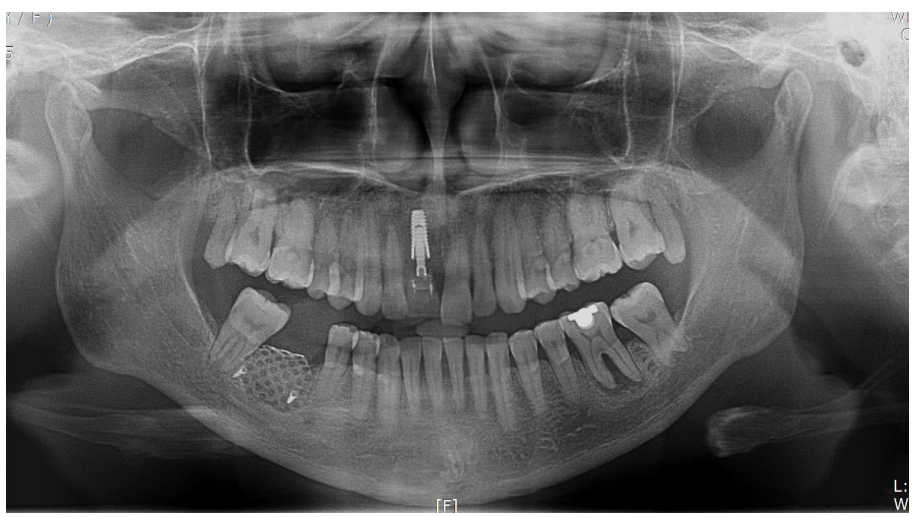

Fig. 3. Case l: Panoramic view after bone grafting with titanium mesh (immediately after the surgery). 

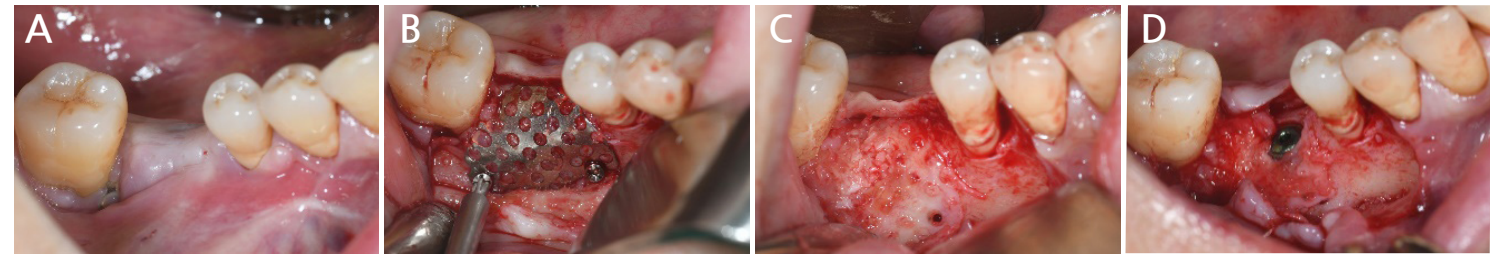

Fig. 4. Case I: Intraoral views after 5 months of bone grafting. (A) Pre-operative intraoral view, (B) A fullthickness flap is elevated and the titanium mesh is removed, (C) The bone augmentation site is healed, (D) The first operative implant surgery.

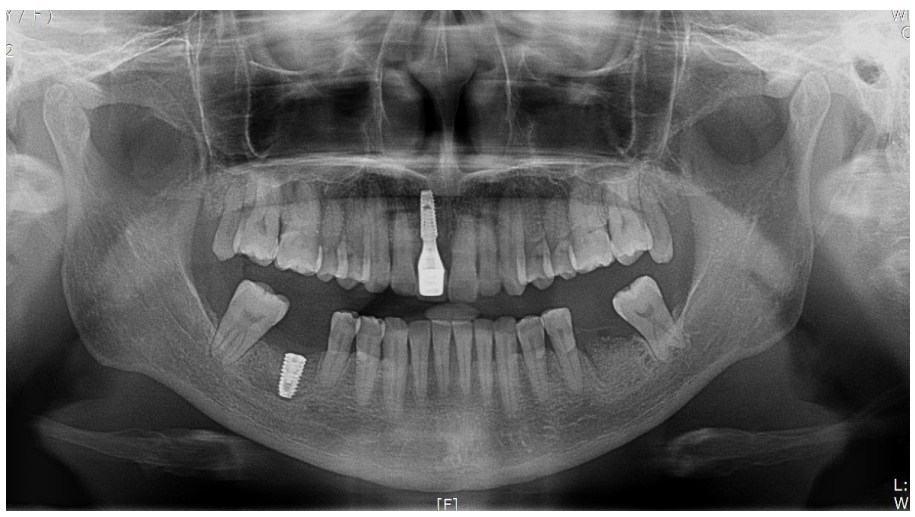

Fig. 5. Case l: Panoramic view after the first operative implant surgery (immediately after the surgery).

well maintained on the panoramic radiograph (Fig. 3). Five months after bone grafting using titanium mesh, the surgical site healed well, and then the first implant placement was performed (Fig. 4A). Similar to the bone graft, crestal and vertical incisions were made bilaterally, and the flap was elevated. The titanium mesh was observed to maintain its shape well, and the micro screw was removed using a screwdriver (Fig. 4B). The grafted bone on the lower side maintained its shape satisfactorily as initially planned, and thus, a sufficient amount of bone was formed (Fig. 4C). Using a general method, an implant with a $4.5 \mathrm{~cm}$ diameter and $8.5 \mathrm{~mm}$ length (TS III ${ }^{\circledR}$; Osstem implant, Seoul, Korea) was placed (Fig. 4D). On the postoperative panoramic radiograph, the implant was observed to be placed satisfactorily at the appropriate position without disturbing the inferior alveolar nerve (Fig. 5).

\section{Case 2}

A 57-year-old male patient was referred to our prosthetics department from a local dental clinic for implant removal and re-implantation due to severe peri-implantitis at the implant site on the left mandible (Fig. 6). Bone augmentation of the vertical and horizontal bone defects (3-4 mm) was performed using a titanium mesh (size $34 \times 25 \mathrm{~mm}$ ) upon removal of the existing implants at the left mandibular first premolar and first molar (Fig. 7).

Two months after implant removal, the removal site healed well. After the crestal incision, the flap was 
elevated, and horizontal and vertical bone defects were observed (Fig. 8A, 8B). Because the existing implant at the adjacent site did not show severe bone loss, it was retained, and bone augmentation was performed using $0.75 \mathrm{cc}$ of allograft bone (Oragraft ${ }^{\circledR}$ ) and a tissue adhesive (fibrin glue) at the bone defect site of each extracted tooth (Fig. 8C). As it would be difficult to maintain the proper bone shape with a bone graft using fibrin glue, titanium mesh was used to maintain the shape and fixed tightly with two micro screws (Fig. 8D). On the panoramic radiograph, bone augmentation using titanium mesh was performed well at the bone defect site of the removed implant, and bone graft in an appropriate shape was observed (Fig. 9). Four months after bone augmentation, the surgical site healed well, and the first

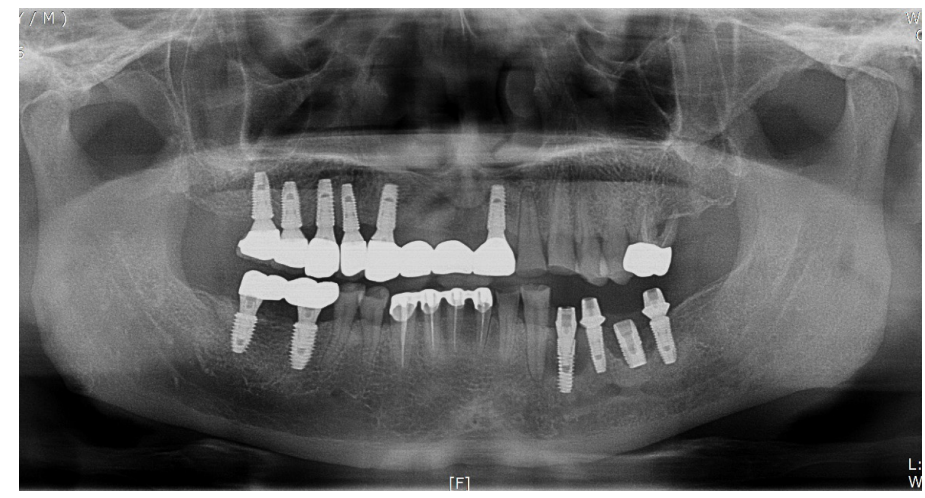

Fig. 6. Case II: Panoramic view at the first visit.

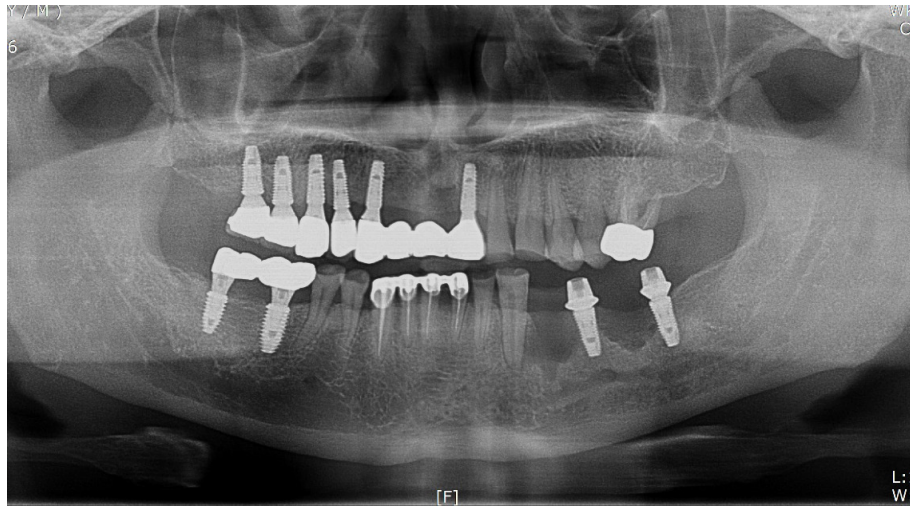

Fig. 7. Case Il: Panoramic view after removal of failed implants.
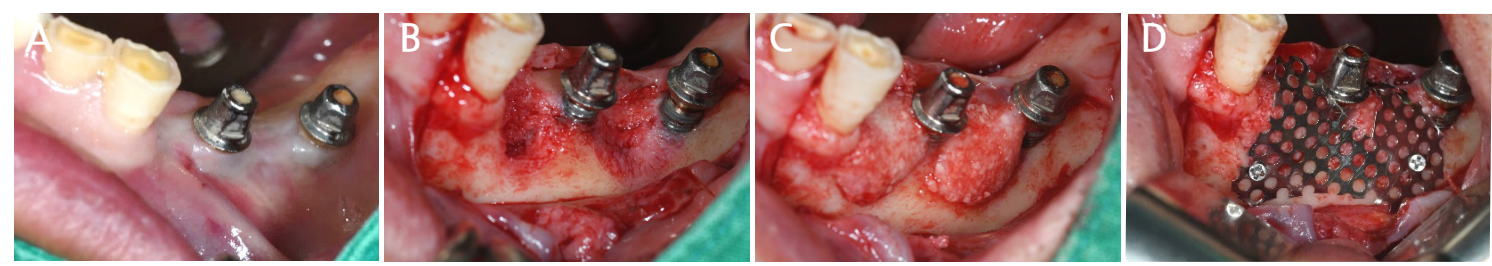

Fig. 8. Case II: Intraoral views of the bone graft with titanium mesh. (A) Pre-operative intraoral view, (B) A full-thickness flap is elevated, (C) Bone grafting using allograft, (D) Application of titanium mesh with micro screws. 
implant surgery was performed (Fig. 10A). After crestal incision and bilateral vertical incisions, the flap was elevated to expose the titanium mesh (Fig. 10B). After the removal of the micro screws, the titanium mesh was removed, and it was confirmed that the planned shape of bone augmentation was achieved. Two implants (Superline ${ }^{\circledR}$; Dentium, Seoul, Korea) with a $4.0 \mathrm{~mm}$ diameter and $10 \mathrm{~mm}$ length were placed in the first premolar and first molar sites, which were the bone graft sites (Fig. 10C, 10D). The postoperative panoramic radiograph showed that bone augmentation was performed well, and the implants were placed at the appropriate positions (Fig. 11).

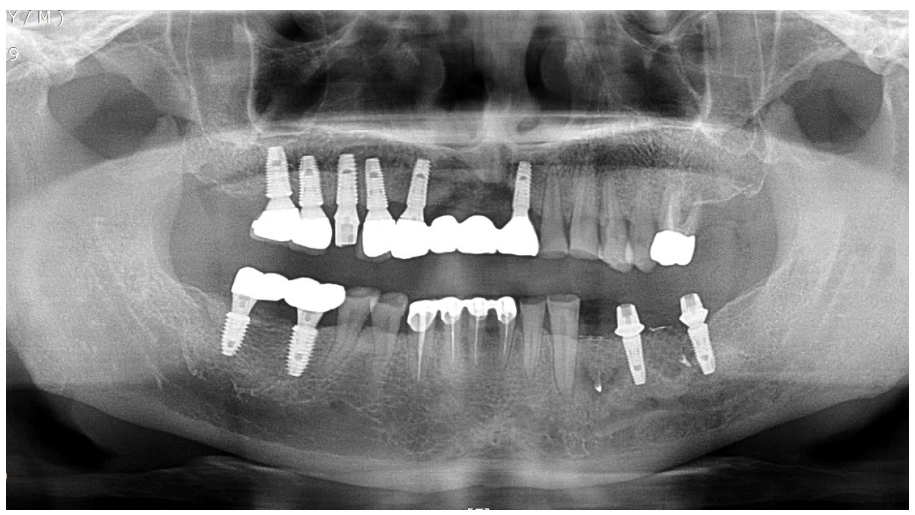

Fig. 9. Case II: Panoramic view after bone graft with titanium mesh (immediately after the surgery).
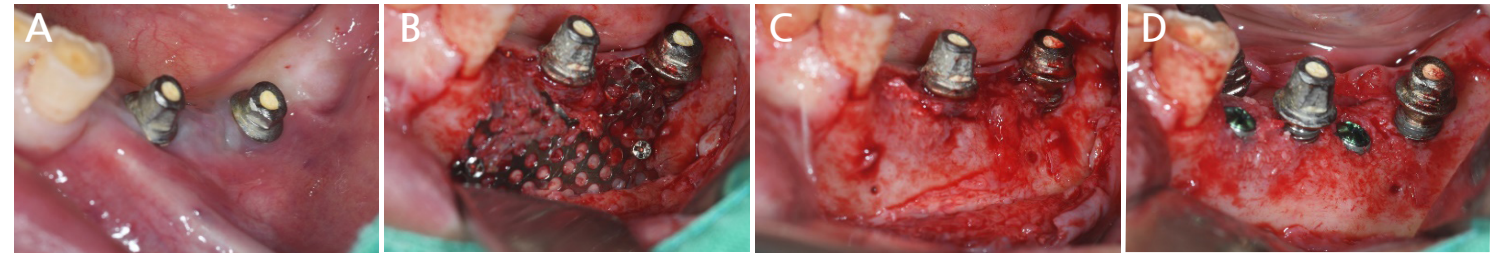

Fig. 10. Case II: Intraoral views after 4 months of bone graft. (A) Pre-operative intraoral view, (B) A fullthickness flap is elevated and the titanium mesh removed, (C) The bone augmentation site is healed, (D) The first operative implant surgery.

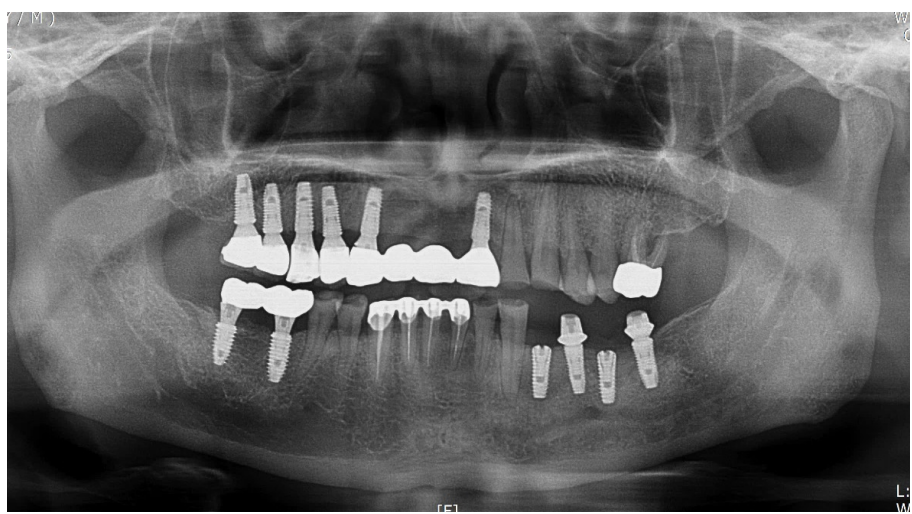

Fig. 11. Case II: Panoramic view after the first operative implant surgery (immediately after the surgery). 


\section{Case 3}

A 42-year-old male patient was referred to our hospital for implant surgery accompanied by a bone graft after tooth extraction due to left mandibular tooth mobility from a local dental clinic. Severe periodontitis of the left mandibular molar site was observed on radiography during the first visit (Fig. 12). Due to severe mobility, bone grafting using titanium mesh was performed after tooth extraction, and implant placement was planned (Fig. 13).

Three months after tooth extraction in the left mandibular molar site, the tooth extraction site healed well (Fig. 14A), and crestal incision was performed under local anesthesia for bone grafting, followed

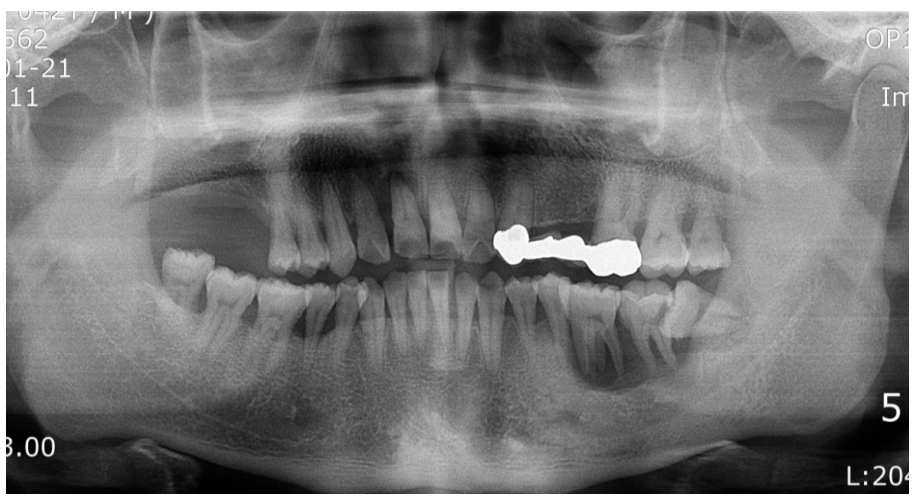

Fig. 12. Case III: Panoramic view at the first visit.

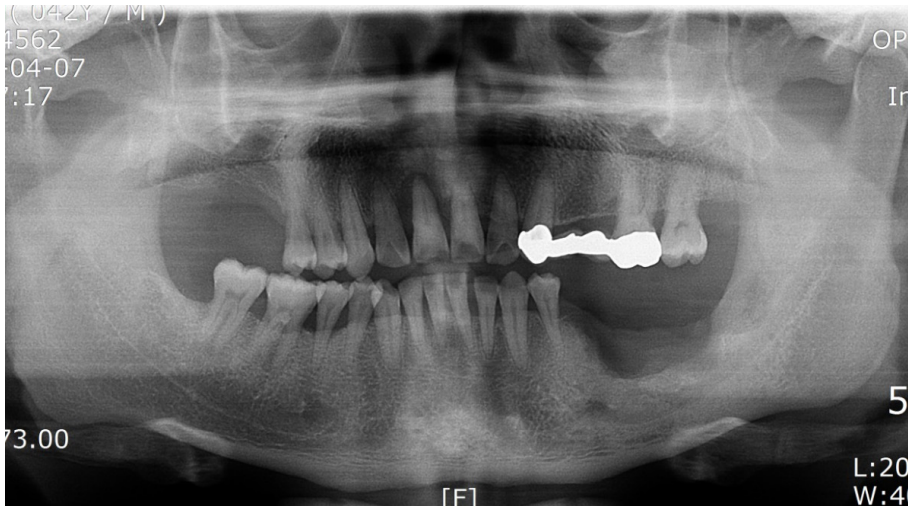

Fig. 13. Case III: Panoramic view after the extraction of teeth.
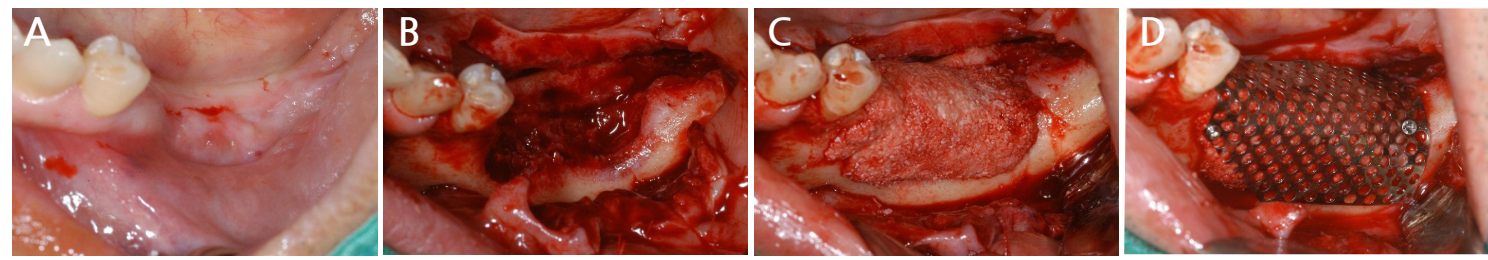

Fig. 14. Case III: Intraoral views of the bone graft with titanium mesh. (A) Pre-operative intraoral view, (B) A full-thickness flap is elevated, (C) Bone graft with allograft and xenograft, (D) Application of titanium mesh with micro screws. 
by elevation of the flap (Fig. 14B). After elevation of the flap, extensive bone destruction (vertically 11 $\mathrm{mm}$, horizontally $32 \mathrm{~mm}$ ) was observed, and bone grafting was performed using allograft bone (Puros ${ }^{\circledR}$; Zimmer Biomet, USA) and xenograft bone (Bio-oss ${ }^{\circledR}$; Geistlich, Wolhusen, Switzerland) with a tissue adhesive (Fig. 14C). After bone grafting, a titanium mesh (size $50 \times 37 \mathrm{~mm}$ ) was used to maintain the shape and space of the vertically grafted bone and fixed well using three micro screws (Fig. 14D). On the postoperative panoramic radiograph, it was found that the bone augmentation using titanium mesh on the site of extensive bone defect in the left mandible had progressed well (Fig. 15).

After 10 months, bone grafting was performed by mixing allograft bone (ICB ${ }^{\circledR}$; Rocky Mountain, Denver, USA) and alloplastic bone (Osteon II $^{\circledR}$; Genoss, Seoul, Korea) at the site where the bone had not formed centrally, and the bone graft site was covered with a resorbable membrane (Collagen membrane; Genoss, Seoul, Korea) and then sutured (Fig. 16). The postoperative panoramic radiograph showed that the bone graft performed well (Fig. 17).

Seven months after the additional bone graft, a crestal incision was made on the surgical site for

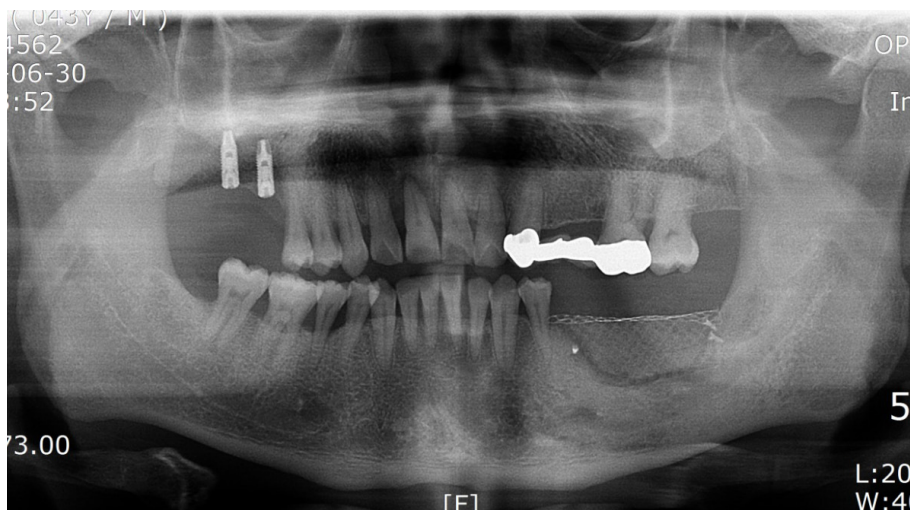

Fig. 15. Case III: Panoramic view after bone graft with titanium mesh (immediately after the surgery).
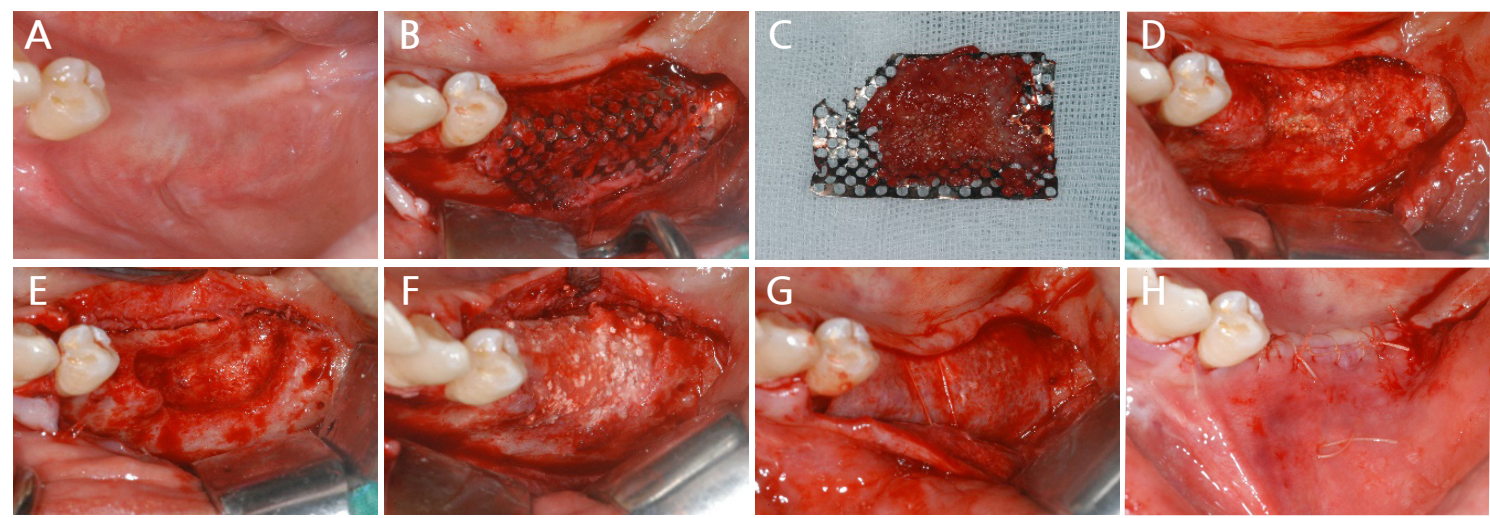

Fig. 16. Case III: Intraoral views after 9 months of bone graft. (A) Pre-operative intraoral view, (B) A fullthickness flap is elevated, (C) Removal of the titanium mesh, (D) The grafted bone is partially infected, (E) Removal of infected bone, (F) Bone graft using allograft and alloplastic bone is performed again, (G) The graft site is covered using a collagen resorbable membrane, $(\mathrm{H})$ The flap is sutured. 
implant placement under local anesthesia, and a flap was elevated to expose the resorbable membrane (Fig. 18A, 18B). When the resorbable membrane was removed, the bone was well-formed at the bone graft site. Subsequently, a total of three implants (Superline ${ }^{\circledR}$; Dentium, Seoul, Korea) were placed: one with a $3.8 \mathrm{~mm}$ diameter and $10 \mathrm{~mm}$ length at the left premolar area, and two with a $4.3 \mathrm{~mm}$ diameter and a $10 \mathrm{~mm}$ length at the left mandibular first and second molar area (Fig. 18C, 18D). Postoperative panoramic radiographs also showed that the implants were well placed at the bone graft site (Fig. 19).

The implant site healed well. After 7 months, the second implant surgery was performed to install a

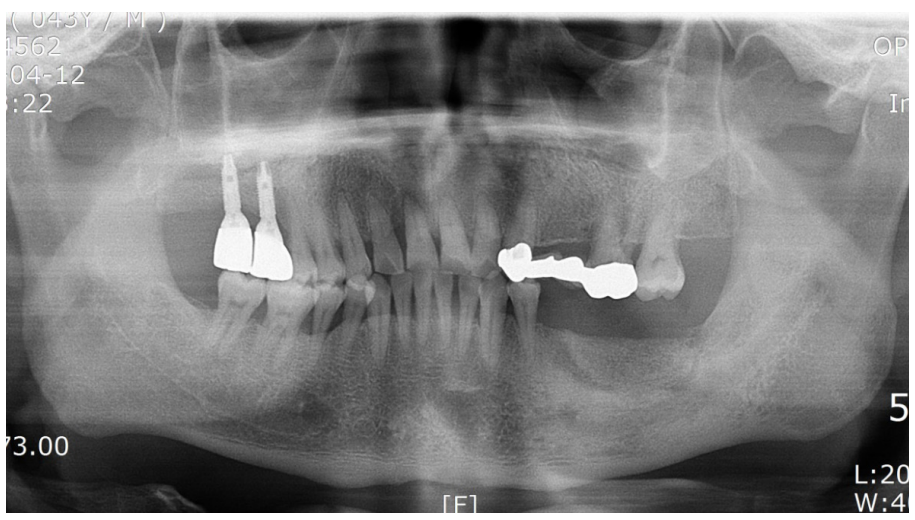

Fig. 17. Case III: Panoramic view after titanium mesh removal and guided bone regeneration (immediately after the procedure).
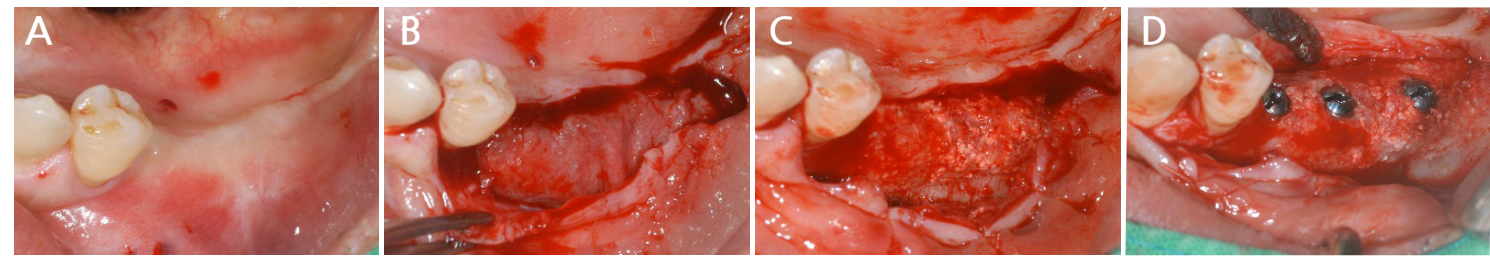

Fig. 18. Case III: Intraoral views after 7 months of guided bone regeneration (GBR). (A) Pre-operative intraoral view, (B) A full-thickness flap is elevated and the resorbable membrane is exposed, (C) The GBR site is healed, (D) First operative implant surgery.

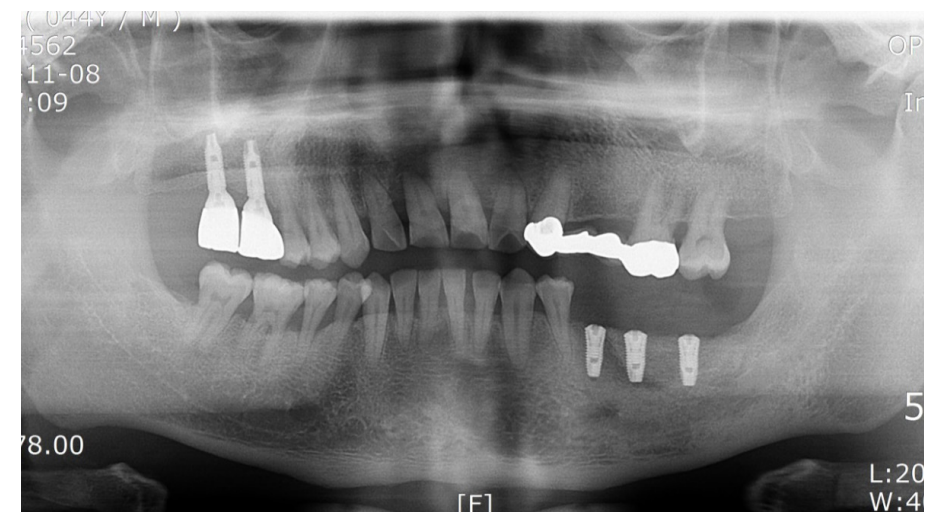

Fig. 19. Case III: Panoramic view after the first operative implant surgery (immediately after the surgery). 
healing abutment, and surgery to expand the attached gingiva zone was also performed (Fig. 20A). After the soft tissue healing was completed following the second surgery, a crown was set, and the attached gingiva formed well (Fig. 20B). After installation of the crown, the grafted bone was observed to be in a good condition on the panoramic radiograph (Fig. 21), and 4 years later, the radiograph showed that the grafted bone was well maintained without loss of the implant marginal bone (Fig. 22).
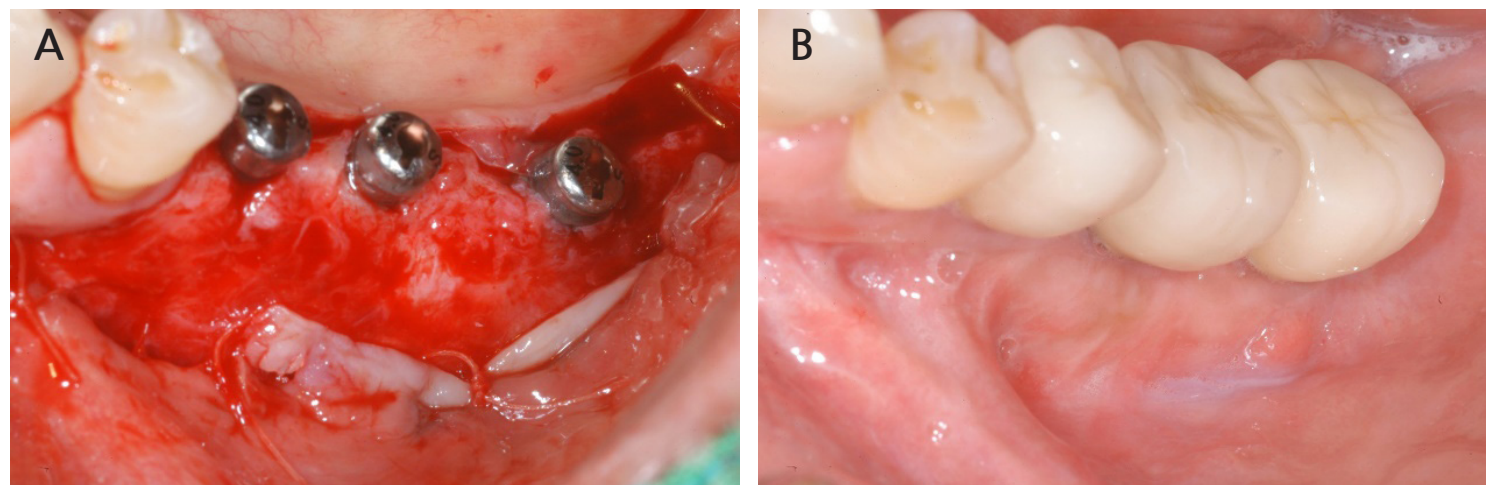

Fig. 20. Case III: Intraoral views of second implant surgery and the final restoration. (A) Intraoral view during surgery, (B) Final restoration setting.

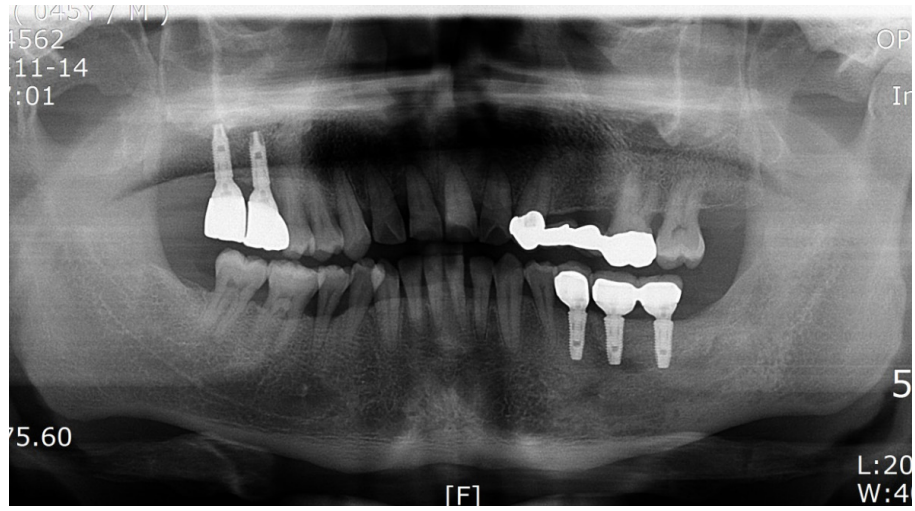

Fig. 21. Case Ill: Panoramic view of the final restoration.

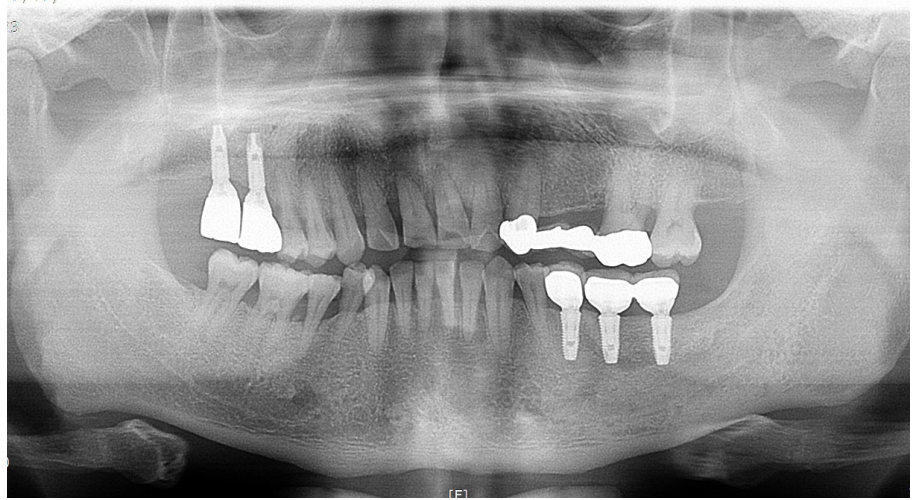

Fig. 22. Case III: Panoramic view of the final restoration after 4 years. 


\section{Discussion}

While various methods are used for bone augmentation when implantation is difficult due to bone defects, GBR using barrier membranes and bone graft materials is widely used owing to its ease of operation and convenience. ${ }^{6}$ However, it is difficult to maintain the shape of the defect site using GBR alone when bone augmentation is performed on a large bone defect, and ensuring vertical formation of the bone is challenging in a vertical bone defect.

As a way to complement the disadvantages of GBR, Simion et al. ${ }^{7}$ in 1994 reported their use of GBR with titanium reinforced Gore-Tex to reconstruct the alveolar ridge with a vertical bone defect, which induced successful bone formation of an average of 3 to $4 \mathrm{~mm}$ after 9 months; since then, this method has been widely used. The non-absorbable Gore-Tex membrane is reinforced with titanium to maintain its shape to a certain extent; however, it also lacks the mechanical strength to create a three-dimensional space, which is insufficient for bone augmentation of the desired shape. In such a case, a procedure that uses a tissue adhesive to immobilize particulate bone graft materials or a titanium mesh to maintain space while maintaining the mechanical strength can be used to form the bone in the desired shape.

Titanium mesh has been used for in vivo three-dimensional reconstruction because it is composed of commercially pure titanium, which is bioinert, easily manipulatable, and has a strong metallic maintenance function. In 2004, Roccuzzo et al. ${ }^{8}$ reported an average bone augmentation effect of 4.8 $\mathrm{mm}$ after vertical bone augmentation was performed with GBR using titanium mesh on the maxillary and mandibular bone defect sites. Additionally, in a 2007 report, a group in which only block bone graft was performed on the bone defect site was compared with that in which augmentation was performed using titanium mesh with bone graft. The results showed that titanium mesh could be effective when vertical bone augmentation was performed, as significantly less bone resorption occurred, and the incidence of complications was also reduced when titanium mesh was used together with the bone graft. $^{9}$

In 2009, Pieri et al. ${ }^{10}$ placed 44 implants after bone grafting using a titanium mesh and bone graft materials for alveolar bone augmentation. It was reported in a 2-year study that all 44 implants showed a 100\% implant survival rate, that is, high survival, and 41 implants were clinically successful, resulting in a 93.1\% success rate. In this case study, bone augmentation was performed using titanium mesh, a non-resorbable membrane, after bone grafting using a tissue adhesive, fibrin glue, at the bone defect site where horizontal and vertical bone resorption had occurred. Titanium mesh with excellent mechanical properties maintained the space of the bone graft site, prevented collapse, and enhanced the stabilization of the wound, thereby enhancing the bone regeneration effect and contributing to the morphological improvement of the regenerated alveolar bone.

Titanium mesh can easily be manipulated according to the shape of the bone defect, and the material 
can resist external forces. However, the barrier membrane can be exposed because of the sharp edges created during cutting and bending, leading to infection and eventual failure of the bone graft. ${ }^{11}$ Additionally, the titanium mesh should be at least $1 \mathrm{~mm}$ away from the site where it meets adjacent teeth, incisions, or a flap to prevent its exposure. In this case study, titanium mesh was also dissected in a curved shape so that there was no sharp edge when it was trimmed, and it was positioned at a sufficient distance from the adjacent incision when applied to the surgical area. In the second case, in which bone augmentation was performed between the tooth and existing implant, the titanium mesh was trimmed carefully to keep the distance between the adjacent tooth and existing implant longer than $1 \mathrm{~mm}$ to prevent exposure of the titanium mesh.

Currently, ready-made titanium mesh products developed by several companies are being marketed. Such pre-made titanium mesh only requires practitioners to select the right product for the bone defect site without the need for trimming; thus, simplifying the surgical process, and solving the problem caused by sharp edges. In all cases, titanium mesh exposure did not occur, and in the first and second cases, a sufficient amount of bone was formed after bone augmentation, allowing implant placement. However, in the third case, the partial bone did not form under the titanium mesh.

In the third case, although there were areas where bone was not formed, a large amount of bone was formed around it. An additional bone graft was performed with GBR using a resorbable membrane, and the implant could be placed 7 months later. In the end, in all the cases using titanium mesh, bone was formed as desired; in particular, vertical bone augmentation was achieved well, allowing the placement of implants of the desired length. In the third case, since attached gingiva did not develop sufficiently after the second surgery, surgery to assist its growth was also performed, followed by prosthetic restoration after soft tissue healing. Four years of observation showed that the grafted bone was well maintained without loss, resulting in satisfactory functional and esthetic outcomes.

\section{Conclusion}

In this clinical case study, bone augmentation of the desired shape was achieved using titanium mesh on the sites of horizontal and vertical bone defects, and implant surgery could be successfully performed.

\section{References}

1. Nyman S. Bone regeneration using the principle of guided tissue regeneration. J Clin Periodontol 1991;18:494-8.

2. Block MS, Degen Y. Horizontal ridge augmentation using human mineralized particulate bone: 
Preliminary results. J Oral Maxillofac Surg 2004;62:67-72.

3. Nyman S, Lang NP, Buser D, Brägger U. Bone regeneration adjacent to titanium dental implants using guided tissue regeneration: a report of two cases. Int J Oral Maxillofac Implants 1990;5:9-14.

4. Buser D, Brägger U, Lang NP, Nyman S. Regeneration and enlargement of jaw bone using guided tissue regeneration. Clin Oral Implants Res 1990;1:22-32.

5. Buser D, Dula K, Belser U, Hirt HP, Berthold H. Localized ridge augmentation using GBR, I. Surgical procedures in the maxilla. Int J Periodontics Restorative Dent 1993;13:29-45.

6. Buser D, Dula K, Hirt HP, Schenk RK. Lateral ridge augmentation using autograts and barrier membranes. A clinical study in 40 partially edentulous patients. Int J Oral Maxillofac Surg 1996;54:420-32.

7. Simion M, Trisi P, Piattelli A. Vertical ridge augmentation using a membrane technique associated with osseointegrated implants. Int J Periodontics Restorative Dent 1994;14:496-511.

8. Roccuzzo M, Ramieri G, Spada MC, Bianchi SD, Berrone S. Vertical alveolar ridge augmentation by means of a titanium mesh and autogenous bone grafts. Clin Oral Implants Res 2004;15:73-81.

9. Roccuzzo M, Ramieri G, Bunino M, Berrone S. Autogenous bone graft alone or associated with titanium mesh for vertical alveolar ridge augmentation: a controlled clinical trial. Clin Oral Implants Res 2007;18:286-94.

10. Pieri F, Corinaldesi G, Fini M, Aldini NN, Giardino R, Marchetti C. Alveolar ridge augmentation with titanium mesh and a combination of autogenous bone and anorganic bovine bone: a 2-year prospective study. J Periodontol 2008;79:2093-103.

11. Her S, Kang T, Fien MJ. Titanium membrane as an alternative to a membrane for ridge augmentation. J Oral Maxillofac Surg 2012;70:803-10. 